\title{
Efficacy of withdrawal time monitoring in adenoma detection with or without the aid of a full-spectrum scope
}

\section{(๑) $\odot \ominus$}

\author{
Authors \\ Institutions \\ 1 Department of Gastroenterology, ASST Rhodense, Rho \\ and Garbagnate Milanese Hospital, Milan, Italy \\ 2 Gastroenterology Unit, ASL Caserta, Marcianise \\ Hospital, Italy \\ 3 Endoscopy Unit, Nuovo Regina Margherita Hospital, \\ Rome, Italy
}

Gianpiero Manes ${ }^{1}$, Paolo Andreozzi ${ }^{2}$, Barbara Omazzi ${ }^{1}$, Cristina Bezzio ${ }^{1}$, Davide Redaelli ${ }^{1}$, Massimo Devani ${ }^{1}$, Daniela Morganti ${ }^{1}$, Raffaella Reati ${ }^{1}$, Simone Saibeni ${ }^{1}$, Enzo Mandelli ${ }^{1}$, Ilaria Arena ${ }^{1}$, Cesare Hassan ${ }^{3}$, Germana de Nucci ${ }^{1}$

submitted 13.9.2018

accepted after revision 22.1.2019

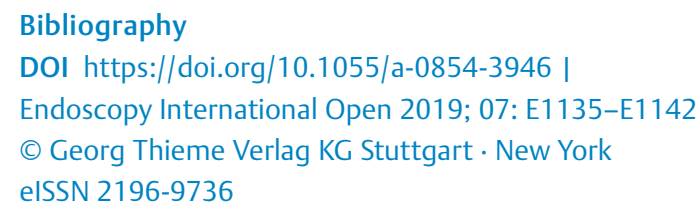

Corresponding author

Gianpiero Manes, MD, Department of Gastroenterology, ASST Rhodense, Viale Forlanini 95, Garbagnate Milanese, Milan, Italy

Fax: +390817463892

gimanes@tin.it

\section{ABSTRACT}

Background and study aims Withdrawal time (WT) monitoring and full-spectrum endoscopy (FUSE) have been suggested to increase adenoma detection rate (ADR) due to more accurate evaluation of the hidden areas of the colon. We aimed to evaluate the efficacy of WT monitoring and FUSE on ADR.

Patients and methods This was a prospective observational study involving consecutive outpatients, aged 18 to 85 years, undergoing colonoscopy with unselected indications. In phase 1 , endoscopists performed 660 colonoscopies either with standard forward-viewing endoscope (SFVE) $(n=330)$ or with FUSE $(n=330)$. In this phase, WTs were measured without endoscopist awareness of being monitored. In phase 2, endoscopists were informed of being monitored and performed additional 660 colonoscopies either with SFVE $(n=330)$ or with FUSE $(n=330)$.

Results WT was lower in phase 1 compared to phase 2 (SFVE: $269 \pm 83$ vs. $386 \pm 60 \mathrm{sec}, P<0.001$; FUSE: $289 \pm 97$ vs. $403 \pm 65 \mathrm{sec}, P<0.001)$. Use of FUSE increased ADR both in phase 1 (33.0\% vs. $27.3 \%, P=0.127)$ and in phase 2 (41.8\% vs. $33.6 \%, P=0.037)$. When endoscopists were aware of being monitored, ADR was higher in SFVE (33.6\% vs. $27.3 \% ; P=0.090)$ and FUSE arms $(41.8 \%$ vs. $33.0 \% ; P=$ $0.024)$. Improvement in detection of proximal adenomas was associated with WT monitoring [OR 1.577 (95\% C.I. $1.158-2.148) ; P=0.004]$, whereas detection of distal adenomas was associated with use of FUSE [OR 1.320 (95\% C. I. $1.022-1.705) ; P=0.037$ ].

Conclusions Unmonitored endoscopists have suboptimal WT, which increases when they are monitored. WT monitoring and use of FUSE are two reliable and alternative strategies to increase ADR.

\section{Introduction}

Screening colonoscopy has been shown to reduce incidence of and mortality from colorectal cancer (CRC) [1, 2]. In spite of recent methodological and technological advancements, colonoscopy remains an imperfect test because up to $27 \%$ of adenomas are missed, which could be responsible for development of a significant percentage of the interval cancers [3]. The colon has several folds and curves that result in hidden areas where small lesions may be not easily visible during colonoscopy. A longer withdrawal time (WT) allows endoscopists to accurately visualize the hidden areas of the colon and to recognize a higher number of lesions. A WT $\geq 6$ minutes has been associated with increased detection of both adenomas and advanced adenomas in several studies [4-6], and it is recommended as a quality indicator in recent European and American guidelines [7]. However, efficacy associated with the implementation of a WT-based policies is still uncertain: prospective interventions directed at optimizing WT yielded conflicting results in terms of ability to increase adenoma detection rate (ADR) [8]. In addition, in randomized back-to-back studies using endoscopes able to enhance the view behind the folds, use of standard 
scopes has been associated with a substantial adenoma miss rate, even though an adequate WT protocol was in place $[9,10]$.

A new scope with a wider angle of view, the full-spectrum endoscope (FUSE, EndoChoice, Alpharetta, Georgia, United States), has been pioneered to increase visibility of hidden areas of the colon [11]. The FUSE has three lenses, one on the forward tip and one on both sides of the tip, that increase the maximum field of view from the $\leq 170^{\circ}$ of standard forwardviewing endoscope (SFVE) to $330^{\circ}$. In theory, use of the FUSE should optimize ADR, irrespective of WT, as its wide angle of view could be a surrogate for the ideal inspection technique that endoscopists try to apply to cover the colonic mucosa when retracting the SFVE. To our knowledge, however, no studies have addressed it until now.

We designed the current study to evaluate whether an intervention directed at optimizing WT alone or in combination with use of the FUSE was able to increase ADR in a cohort of outpatient colonoscopies. In other words, we wanted to understand whether use of the FUSE would further increase ADR, once WT had been optimized.

\section{Patients and methods}

\section{Study design and population}

This was a prospective, observational study performed in consecutive outpatients scheduled for elective colonoscopy during a 6-months period (June 2016 - December 2016). The study was conducted in two high-volume endoscopic centers belonging to the same Gastroenterology Department. The study protocol was approved by the local ethics committee and was registered at ClinicalTrials.gov (NCT02985944). Written informed consent was obtained from all enrolled patients.

Inclusion criteria for enrollment were referral for diagnostic examination, age 18 to 85 years, and written informed consent provided. Patients were excluded if they were hospital inpatients, had a history of colorectal resection or of recent major abdominal surgery, had previously been diagnosed with a polyposis syndrome, or had inflammatory bowel disease. Patients with incomplete colonoscopy were also excluded.

\section{Study design}

The study was designed within a quality audit program that began with introduction of the new FUSE platform in the two Endoscopic Units. During the study period, the endoscopists were not aware that a study was running. In the first 3-month period, six expert endoscopists performed colonoscopy either with high-definition standard forward-viewing endoscopy (SFVE) or with FUSE, according to local availability. In the first phase (phase 1) of the study, colonoscopies were performed while a nurse monitored WT with the endoscopists who were unaware of WT monitoring. During a subsequent 3-month period (phase 2) the same endoscopists performed additional colonoscopies either with high-definition SFVE or with FUSE, but they were informed that their WT would be monitored by a nurse to check the performance of each endoscopist with the new FUSE scope in comparison with the old SFVE ( $\triangleright$ Fig. 1).
In both phases, assignment of the patients to undergo colonoscopy with SFVE or FUSE was determined by scope availabilit in the endoscopic suite so that the patients underwent colonoscopy with the one or the other scope at a 1:1 ratio. Because the endoscopists could not be blinded to scope type, and no differences were expected in terms of age, sex, and indication, a formal randomization process was not believed to improve the validity of the study findings.

\section{Examination procedure}

Patients underwent bowel cleansing with $2 \mathrm{~L}$ of polyethylene glycol solution plus bisacodyl in a split-dose or day-before regimen according to the scheduled time of colonoscopy. Participants in both groups were instructed to have a light lunch on the day before the colonoscopy; only clear liquid were allowed the day of the exam. All endoscopic procedures were performed between 9 am and 2 pm.

Six experienced endoscopists (>1000 standard colonoscopies) performed the endoscopic procedures in accordance with colonoscopy quality practice. If not contraindicated, endoscopies were performed using intravenous sedation with midazolam and/or fentanyl.

Colonoscopy was defined as complete when the appendix orifice and the ileocecal valve were identified. Bowel preparation was evaluated by using the Boston Bowel Preparation Scale (BBPS) score as previously reported [10]. Preparation was considered adequate when the BBPS score was $\geq 6$ with a score of at least two in any segments.

SFVE was performed by using high-definition, white-light, adult colonoscope (Pentax 90 I series HD Video colonoscopes). Full-spectrum colonoscopy was performed by using the new high-definition FUSE system (FUSE, EndoChoice, Alpharetta, Georgia, United States) consisting of a high-definition processor and an adult colonoscope with a 330-degree field of view. Images of colonic mucosa were displayed on three contiguous screens.

All endoscopists had been trained to use the FUSE system before study initiation by attending a lecture and performing at least 10 examinations with the FUSE.

\section{Withdrawal time assessment}

Colonoscopy WT was defined as time taken to withdraw the colonoscope from the cecum to the anus. Time of endoscopic procedures such as polypectomy or taking a biopsy, as well as washing and sucking, was not included in the calculation of WT.

In the first phase of the study, a trained nurse monitored colonoscopy WT using a stopwatch, while the endoscopists were unaware that WT was being monitored. In the second phase of the study, the endoscopists were informed of the WT monitoring.

\section{Assessment}

For each patient, demographic and clinical characteristics, indications for colonoscopy, and colonoscopy characteristics (i.e. quality of colon preparation, cecal intubation time, WT, and endoscopic findings) were collected. All polyps were removed 


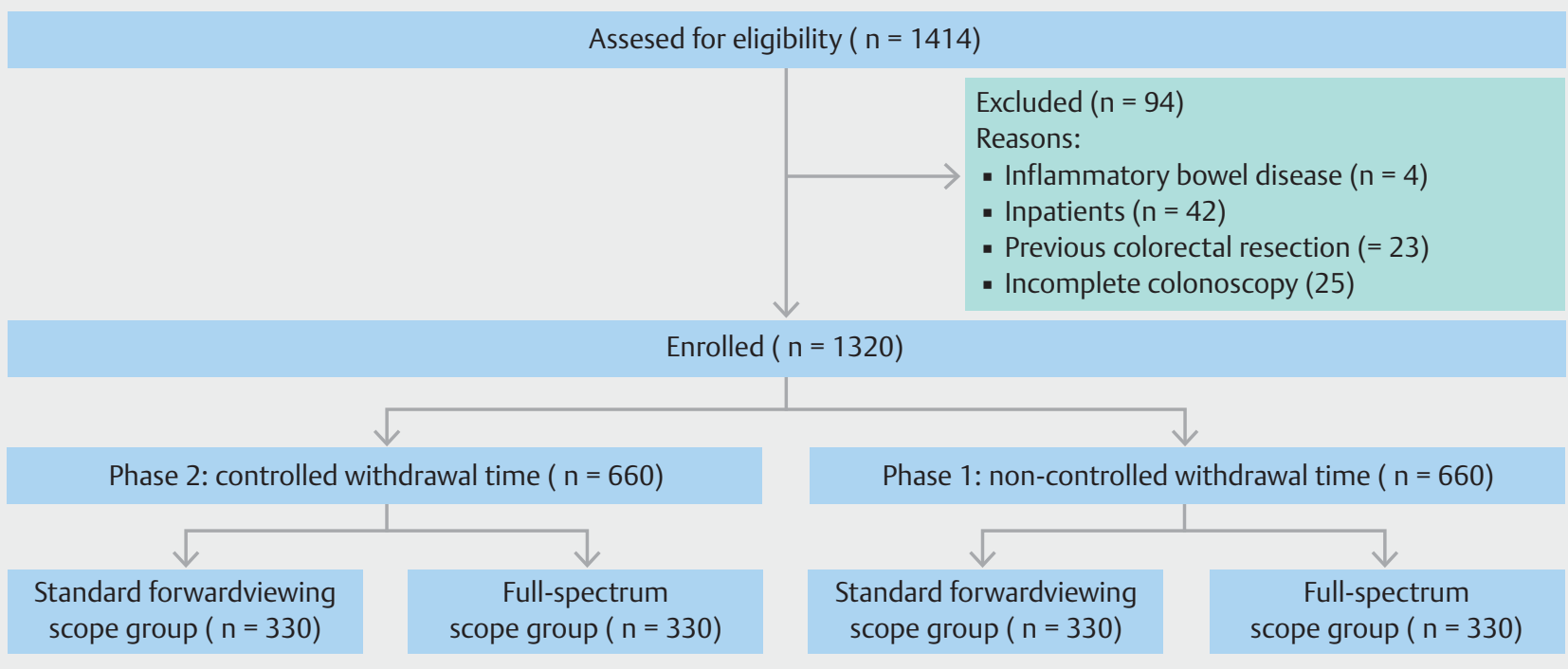

Fig. 1 Flowchart of the study.

and sent to the pathologist. Polyps were described and classified according to their size, location, and morphology.

For each arm, we calculated the mean number of adenomas per colonoscopy (APC) and the ADR, i.e. the proportion of patients with at least one adenoma. Sessile serrated adenomas/ polyps were included in the calculation of ADR.

The primary endpoint of the study was the ADR, whereas APC and rate of adenomas located proximally or distally to the splenic flexure represented a secondary endpoint of the study.

\section{Sample size and statistical analysis}

The endoscopy database of our centers showed an observed prevalence of adenomas of $24 \%$ in patients undergoing screening, surveillance or diagnostic colonoscopy. A previous study reported an ADR of $36 \%$ when WT was monitored [10]. Based on these data, the sample size needed to show a significant difference between the SFVE and FUSE groups at the 0.05 alpha level with a power of $90 \%$ would be 304 per group.

Categorical variables and continuous variables were expressed as percentages and means \pm standard deviations, respectively. Pearson's chi-squared test and Fisher's exact test were used for the categorical variables where appropriate. Student's t-test and ANOVA test were used to evaluate differences in continuous variables among the groups.

Binary backward stepwise logistic regression was performed with "withdrawal time $\geq 6$ minutes" as binary dependent variable in phase 1 (the endoscopists were unaware of the WT being monitored). Covariates included patient age (over 50 vs. under 50 years), sex (male vs. female), adequate bowel cleansing (yes vs. no), bleeding (yes vs. no), gastrointestinal symptoms (yes vs. no), colorectal cancer screening (yes vs. no), adenoma surveillance (yes vs. no), detection of an adenoma during the examination (yes vs. no), use of the FUSE (yes vs. no) and adenoma detection during the procedure (yes vs. no). Effect size was expressed as odds ratios with $95 \%$ confidence intervals $(\mathrm{Cl})$.

Data analysis was performed by using IBM SPSS, version 20.0 and the statistical significance was set at $P<0.05$.

\section{Results}

\section{Patient characteristics}

A flow diagram of study design is shown in > Fig. 1. In the two 3-month study periods, 1414 patients were considered for enrollment and 94 patients were excluded because of violation of inclusion and exclusion criteria. The remaining 1320 patients were included in the study, 660 patients in phase 1 and 660 in phase 2 . In each phase of the study, 330 patients were allocated into the SFVE group and 330 patients into the FUSE group.

- Table 1 shows characteristics of the participants in the four arms of the study. No significant differences in demographic characteristics of patients and indications for colonoscopy were observed among groups.

\section{Colonoscopy characteristics}

- Table 2 lists characteristics of the colonoscopy procedures. No difference in rate of adequate cleansing was observed among the different arms. Cecal intubation time was significantly longer in the FUSE arms compared to the SFVE arms in both phases (phase 1: $379 \pm 182$ vs. $340 \pm 164$ sec, $P=0.026$; phase 2: $383 \pm 160$ vs. $334 \pm 220 \mathrm{sec}, P=0.003$; $/$ Fig. 2a). Overall, WT was shorter in phase 1 compared to phase 2 (SFVE: 269 \pm 83 vs. $386 \pm 60 \mathrm{sec}, P<0.001$; FUSE: $289 \pm 97$ vs. $403 \pm 65 \mathrm{sec}$, $P<0.001$; Fig. 2b) and was longer for the FUSE in comparison to SFVE (phase 1: $289 \pm 97$ vs. $269 \pm 83 \mathrm{sec}, P=0.006$; phase 2: $403 \pm 65$ vs. $386 \pm 60 \mathrm{sec}, P<0.020)$. WT $\geq 6$ minutes was observed in 151 colonoscopies in phase 1 and 587 colonoscopies in phase $2(22.9 \%$ vs. $88.9 \%, P<0.001)$. 
- Table 1 Demographics and clinical features of the different study groups.

\begin{tabular}{|c|c|c|c|c|c|}
\hline & \multicolumn{2}{|l|}{ Phase 1} & \multicolumn{2}{|l|}{ Phase 2} & \multirow[t]{2}{*}{$P$ value } \\
\hline & $\begin{array}{l}\text { SFVE } \\
(n=330)\end{array}$ & $\begin{array}{l}\text { FUSE } \\
(n=330)\end{array}$ & $\begin{array}{l}\text { SFVE } \\
(n=330)\end{array}$ & $\begin{array}{l}\text { FUSE } \\
(n=330)\end{array}$ & \\
\hline Age (yrs), mean $\pm S D$ & $60.5 \pm 12.5$ & $59.5 \pm 13.8$ & $59.6 \pm 12.8$ & $60.5 \pm 13.6$ & 0.674 \\
\hline Male sex, \% (n) & $178(53.9)$ & $182(55.2)$ & $181(54.8)$ & $185(56.1)$ & 0.959 \\
\hline \multicolumn{6}{|l|}{ Indications, \% ( $n$ ) } \\
\hline Screening & $29.7 \%(98)$ & $27.9 \%(92)$ & $29.1 \%(96)$ & $34.8 \%(115)$ & 0.219 \\
\hline Bleeding & $22.7 \%(75)$ & $25.2 \%(83)$ & $22.4 \%(74)$ & $23.9 \%(79)$ & 0.837 \\
\hline Post-polypectomy & $22.1 \%(73)$ & $22.7 \%(75)$ & $22.7 \%(75)$ & $15.5 \%(51)$ & 0.056 \\
\hline Gastrointestinal symptoms & $25.5 \%(84)$ & $24.2 \%(80)$ & $25.8 \%(85)$ & $25.8 \%(85)$ & 0.965 \\
\hline
\end{tabular}

Table 2 Characteristics of colonoscopy procedures.

\begin{tabular}{|c|c|c|c|c|c|c|c|}
\hline & \multicolumn{3}{|l|}{ Phase 1} & \multicolumn{4}{|l|}{ Phase 2} \\
\hline & $\begin{array}{l}\text { SFVE } \\
(n=330)\end{array}$ & $\begin{array}{l}\text { FUSE } \\
(n=330)\end{array}$ & P value ${ }^{1}$ & $\begin{array}{l}\text { SFVE } \\
(n=330)\end{array}$ & $\begin{array}{l}\text { FUSE } \\
(n=330)\end{array}$ & $P$ value $^{2}$ & $\begin{array}{l}\text { Overall } \\
\text { Pvalue }^{3}\end{array}$ \\
\hline Adequate cleansing, \% ( $\mathrm{n}$ ) & $86.4 \%(285)$ & $85.2 \%(281)$ & 0.738 & $89.1 \%(294)$ & $88.5 \%(292)$ & 0.805 & 0.391 \\
\hline Cecal intubation time (sec), mean \pm SD & $340 \pm 164$ & $379 \pm 182$ & 0.026 & $334 \pm 220$ & $383 \pm 160$ & $<0.01$ & $<0.01$ \\
\hline Withdrawal time (sec), mean \pm SD & $269 \pm 83$ & $289 \pm 97$ & $<0.01$ & $386 \pm 60$ & $403 \pm 65$ & 0.020 & $<0.01$ \\
\hline Withdrawal time $\geq 6 \min , \%$ ( $n$ ) & $17.0 \%(56)$ & $28.8 \%(95)$ & $<0.01$ & $92.4 \%(305)$ & $85.5 \%(282)$ & $<0.01$ & $<0.01$ \\
\hline $\mathrm{ADR}, \%(\mathrm{n})$ & $27.3 \%(90)$ & $33.0 \%(109)$ & 0.127 & $33.6 \%(111)$ & $41.8 \%(138)$ & 0.037 & 0.001 \\
\hline $\mathrm{APC}$, mean $\pm \mathrm{SD}$ & $0.43 \pm 0.85$ & $0.56 \pm 1.08$ & 0.071 & $0.65 \pm 1.24$ & $0.71 \pm 1.08$ & 0.502 & 0.004 \\
\hline Right colon ADR, \% (n) & $11.2 \%(37)$ & $12.7 \%(42)$ & 0.632 & $16.4 \%(54)$ & $18.9 \%(62)$ & 0.415 & 0.023 \\
\hline Left colon ADR, \% ( $n$ ) & $20.0 \%(66)$ & $24.8 \%(82)$ & 0.081 & $21.9 \%(72)$ & $27.0 \%(89)$ & 0.147 & 0.153 \\
\hline$\leq 5 \mathrm{~mm} \mathrm{ADR}, \%(\mathrm{n})$ & $18.2 \%(60)$ & $21.5 \%(71)$ & 0.329 & $24.5 \%(81)$ & $37.0 \%(122)$ & $<0.01$ & $<0.001$ \\
\hline $6-9 \mathrm{~mm}$ ADR, \% (n) & $7.9 \%(26)$ & $9.1 \%(30)$ & 0.675 & $11.8 \%(39)$ & $9.4 \%(31)$ & 0.376 & 0.373 \\
\hline$>10 \mathrm{~mm}$ ADR, \% (n) & $5.5 \%(18)$ & $8.5 \%(28)$ & 0.168 & $9.1 \%(30)$ & $7.0 \%(23)$ & 0.390 & 0.285 \\
\hline $\begin{array}{l}\text { SFVE, standard forward-viewing endoscope } \\
1 P \text { value SFVE vs FUSE (phase } 1 \text { ) } \\
2 P \text { value SFVE vs FUSE (phase } 2 \text { ) } \\
{ }^{2} P \text { value all groups }\end{array}$ & USE, full-spectru & ndoscope; ADR, & noma detect & n rate; APC, ader & ma per colonosc & & \\
\hline
\end{tabular}

\section{Effect of WT and endoscope type on ADR}

Overall, 1110 polyps were detected, 779 of which were adenomas.

In phase 1, ADR was higher when endoscopists used the FUSE, but this difference was not statistically significant (33.0\% vs. $27.3 \%$; $P=0.127$ ). In phase 2 , ADR was significantly higher for the FUSE than for SFVE $(41.8 \%$ vs. $33.6 \%, P=0.037)$. When endoscopists were aware of being monitored, ADR increased both for SFVE (33.6\% vs. $27.3 \% ; P=0.090)$ and FUSE (41.8\% vs. $33.0 \%$; $P=0.024)$. Overall, when endoscopists were aware of being monitored and used the FUSE, ADR resulted to be significantly higher in comparison to other arms of the study ( $\triangleright$ Fig. 3a and $\triangleright$ Table 2 ).
Similar results were observed for mean APC, which was lower in the SFVE arm of phase 1 compared to the SFVE and FUSE arms of phase $2(0.43 \pm 0.85$ vs $0.65 \pm 1.24, P=0.040 ; 0.43 \pm$ 0.85 vs. $0.71 \pm 1.08, P=0.003$ )( $($ Table 2 ).

The ADR for adenomas located proximally to the splenic flexure was higher when endoscopists were aware of being monitored (SFVE arms: $11.2 \%$ vs. $16.4 \%, P=0.056$; FUSE arms: $12.7 \%$ vs. $18.9 \%, P=0.033)$. Use of the FUSE added only a little to the diagnostic gain obtained by SFVE after optimization of WT (SFVE $16.4 \%$ vs FUSE 18.9\%) ( Fig. 3 b and $>$ Table 2 ). Conversely, the ADR for adenomas located distally to the splenic flexure tended to be higher in the FUSE arms compared to SFVE arms, although these differences were not statistically 


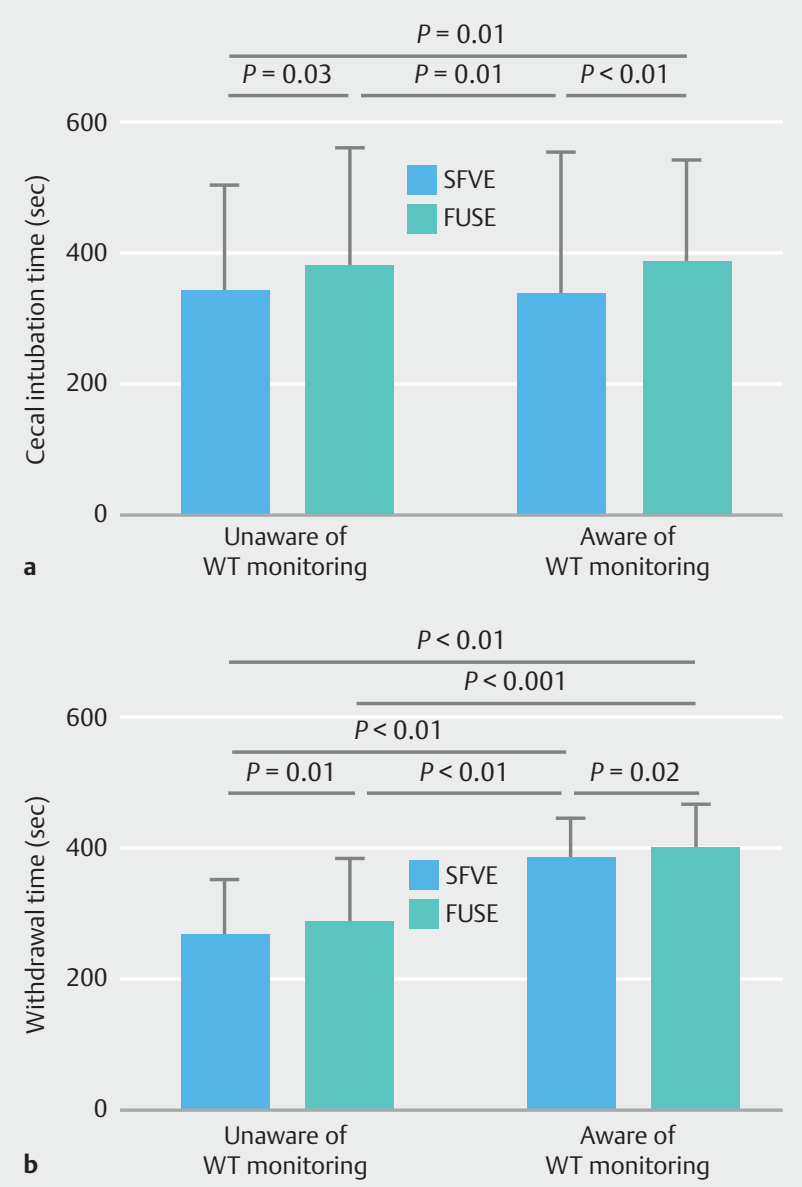

- Fig.2 Cecal intubation time and withdrawal time in the two phases of the study.

significant (phase $1: 20.0 \%$ vs. $24.8 \%, P=0.081$; phase $2: 21.8 \%$ vs. $27.0 \%, P=0.147)(\triangleright$ Fig. 3 c and $\triangleright$ Table 2 ).

Improvement in the diagnostic gain obtained by optimizing WT and using the FUSE was seen only for adenomas $\leq 5 \mathrm{~mm}$ while no differences were found in ADR for adenomas $\geq 6 \mathrm{~mm}$ among the different arms of the study ( $\bullet$ Table 2 ).

Overall on univariate analysis, both WT monitoring [OR 1.403 (95\% C.I. $1.116-1.765) ; P=0.004]$ and use of a FUSE [OR 1.366 (95\% C.I. 1.086-1.717); $P=0.009$ ] positively influenced ADR. In particular, detection of proximal adenoma was associated with WT monitoring [OR 1.577 (95\% C.I. 1.158 2.148); $P=0.004$ ], whereas detection of distal adenoma was associated with use of the FUSE [OR 1.320 (95\% C.I. 1.022 1.705); $P=0.037$ ] ( Fig. 4).

On multivariate analysis, patients aged older than 50 years, colorectal cancer (CRC) screening as indication to colonoscopy, and adequate bowel cleansing were positively associated witho detection of at least one adenoma. Also WT monitoring and use of the FUSE resulted in a significantly higher ADR. Conversely, female sex and other indications for colonoscopy (bleeding, adenoma surveillance, gastrointestinal symptoms) were negatively associated with adenoma detection ( $\triangleright$ Table 3 ).

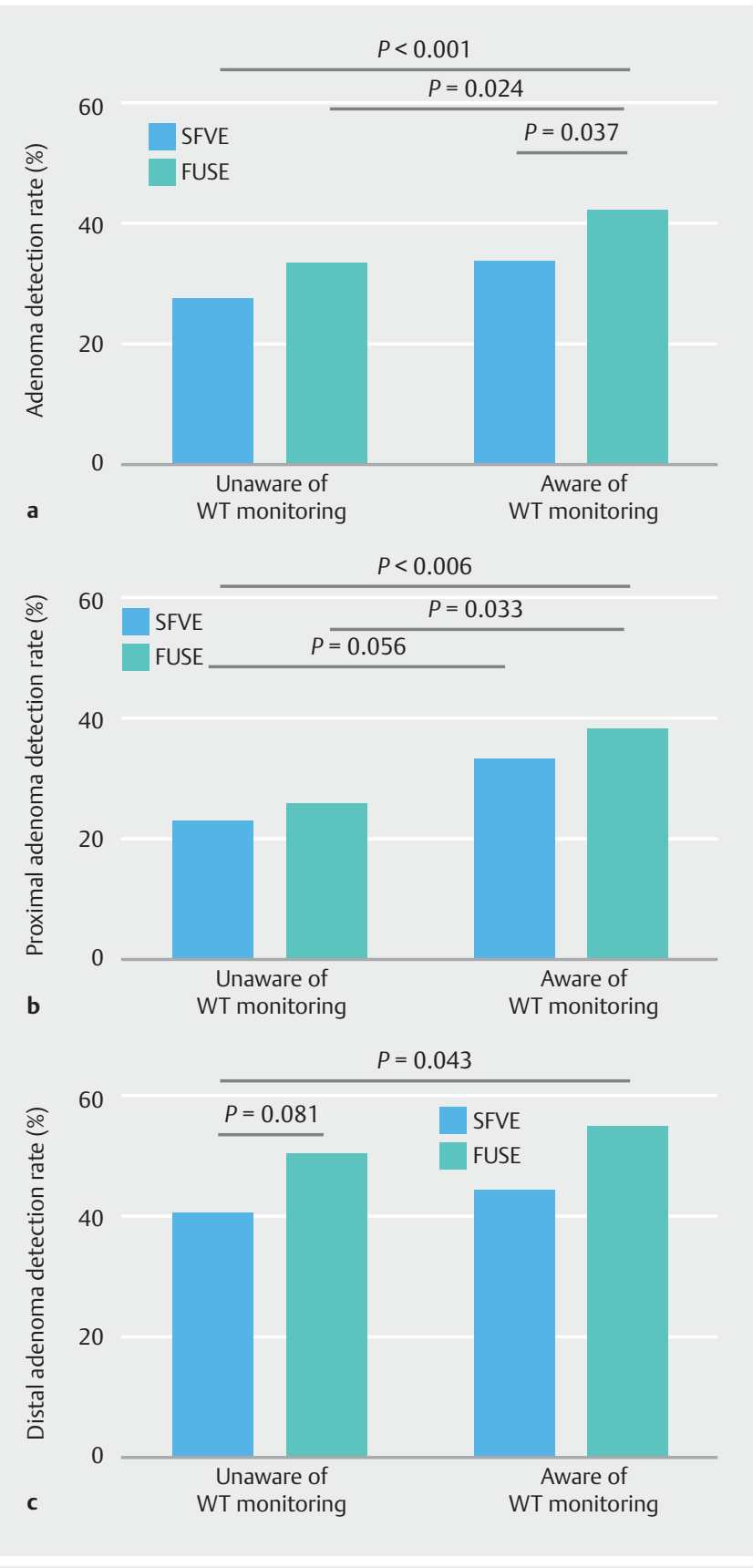

Fig. 3 Adenoma detection rate in the different study arms.

\section{Factors affecting WT}

In phase 1 of the study, WT was affected by several factors $(\vee$ Table 4). On multivariate analysis, detection of a first adenoma during the examination, adequate bowel cleansing, colonoscopy performed for CRC screening, and use of the FUSE were positively associated with $W T \geq 6$ minutes, whereas female sex was negatively associated with $W T \geq 6$ minutes. In phase 2 , the only factor that was positively associated with $W T \geq 6$ minutes was detection of a first adenoma during the procedure (data not shown). 


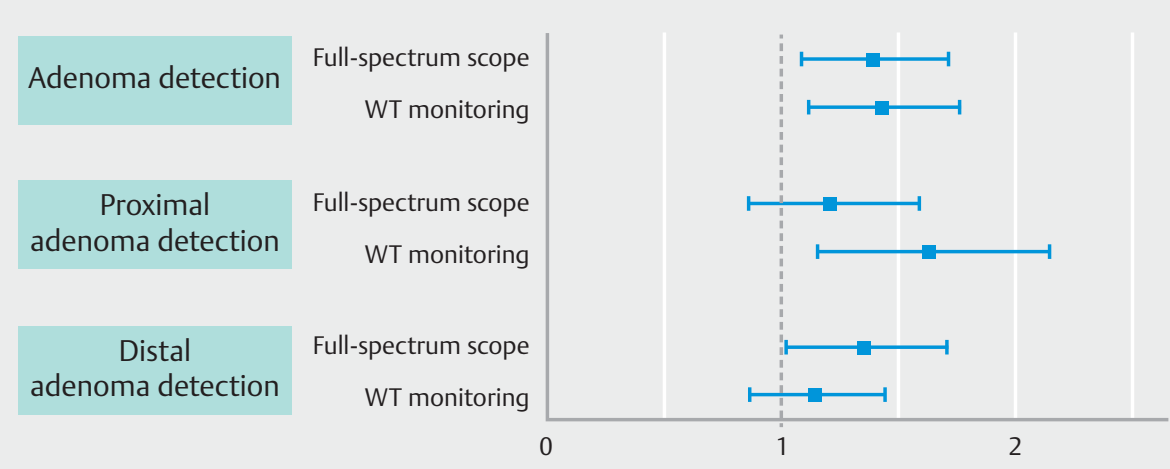

Fig. 4 Effect of SFVE and FUSE on rate of detection of adenomas according to their position (proximal or distal to the splenic flexure).

- Table 3 Univariate and multivariate logistic regression model for adenoma detection.

\begin{tabular}{|l|l|l|l|}
\hline Binary outcome "detection of at least one adenoma" (yes vs. no) & & & \\
\hline & $\begin{array}{l}\text { Univariate model } \\
\text { Odds ratio (95\%CL) }\end{array}$ & P value & $\begin{array}{l}\text { Multivariate model } \\
\text { Odds ratio (95\%CL) }\end{array}$ \\
\hline Female sex & $0.400(0.314-0.509)$ & $<0.001$ & $0.397(0.308-0.511)$ \\
\hline Age over 50 years & $4.192(2.925-6.007)$ & $<0.001$ & $4.204(2.890-6.116)$ \\
\hline Adequate bowel cleansing & $1.471(1.024-2.113)$ & 0.037 & $1.597(1.091-2.338)$ \\
\hline Bleeding & $0.726(0.550-0.958)$ & 0.024 & $0.579(0.414-0.808)$ \\
\hline CRC screening & $1.861(1.460-2.372)$ & $<0.001$ & $1.728(1.238-2.414)$ \\
\hline Adenoma surveillance & $1.107(0.838-1.463)$ & 0.474 & $0.635(0.455-0.885)$ \\
\hline Gastrointestinal symptoms & $0.586(0.444-0.774)$ & $<0.001$ & $0.594(0.424-0.832)$ \\
\hline Use of full-spectrum scopes & $1.366(1.086-1.717)$ & 0.009 & $1.425(1.131-1.843)$ \\
\hline Withdrawal time monitoring & $1.403(1.116-1.765)$ & 0.004 & $1.444(1.117-1.819)$ \\
\hline CRC, colorectal cancer. & & 0.0016 \\
\hline
\end{tabular}

- Table 4 Factors affecting the withdrawal time in the phase 1 of the study: univariate model and multivariate logistic regression model for withdrawal time $\geq 6$ minutes.

\begin{tabular}{|c|c|c|c|c|}
\hline \multicolumn{5}{|c|}{ Binary outcome "withdrawal time $\geq 6$ min" (yes vs. no) } \\
\hline & $\begin{array}{l}\text { Univariate model } \\
\text { Odds ratio }(95 \% \mathrm{Cl})\end{array}$ & $P$ value & $\begin{array}{l}\text { Multivariate model } \\
\text { Odds ratio }(95 \% \mathrm{Cl})\end{array}$ & $P$ value \\
\hline Female sex & $0.331(0.221-0.495)$ & $<0.001$ & $0.416(0.270-0.643)$ & $<0.001$ \\
\hline Age over 50 years & $1.562(0.964-2.532)$ & 0.071 & $0.970(0.564-1.669)$ & 0.913 \\
\hline Adequate bowel cleansing & $2.009(1.085-3.720)$ & 0.024 & $2.162(1.126-4.152)$ & 0.021 \\
\hline Adenoma detection & $3.693(2.526-5.399)$ & $<0.001$ & $2.466(1.626-3.740)$ & $<0.001$ \\
\hline Bleeding & $0.561(0.350-0.900)$ & 0.017 & $0.612(0.370-1.012)$ & 0.056 \\
\hline CRC Screening & $2.909(1.989-4.252)$ & $<0.001$ & 2.6981 .5454 .714 & $<0.001$ \\
\hline Adenoma surveillance & $1.110(0.723-1.704)$ & 0.657 & $1.512(0.823-2.779)$ & 0.183 \\
\hline Gastrointestinal symptoms & $0.361(0.215-0.607)$ & $<0.001$ & $0.815(0.418-1.589)$ & 0.548 \\
\hline Use of full-spectrum scopes & $1.978(1.362-2.873)$ & $<0.001$ & $2.039(1.361-3.053)$ & 0.001 \\
\hline
\end{tabular}




\section{Discussion}

Our study demonstrates that combining WT monitoring and use of a wide-angle scope results in a clinically relevant increase in number of lesions detected. Several studies have demonstrated the positive association between ADR and/or APC and WT $[4,12]$, but, to the best of our knowledge, this is the first study designed with the aim of understanding whether use of the FUSE results in further diagnostic gain once WT and withdrawal technique have been optimized.

Longer WT allows for more accurate visualization of the back of the folds and inner curve of the flexures, which are usually not easily visible when using a SFVE. However, even when used with an optimal withdrawal technique, SFVE is likely to be unable to detect some polyps. Evidence from colonoscopy tandem studies indicates that SFVE may have a higher adenoma miss rate when compared with systems that potentially enable visualization of mucosa behind the folds, such as the FUSE and EndoRing [9, 13].

Moreover, some studies have demonstrated that prospective institution of an optimal WT of at least 7 minutes may have no impact on polyp detection rates [8]; similarly, a prospective cohort study determined that WT, using 6 minutes as the threshold, was not a strong predictor of likelihood of finding a polyp [14]. All these data are likely to suggest that SFVE may have intrinsic limitations in visualizing the entire colonic mucosa that cannot be overcome by prolonging inspection time.

The FUSE system has been recently introduced to overcome intrinsic limitations in the SFVE. In phase 1 of our study, when both the FUSE and SFVE were used at a suboptimal WT, the FUSE enabled an increase in ADR from $27.3 \%$ to $33 \%$ in comparison with SFVE; in phase 2, accuracy of both SFVE and FUSE increased, but the FUSE again allowed detection of more adenomas, increasing ADR from $33.6 \%$ to $41.8 \%$. That means that the vast majority of lesions can be detected by SFVE using a slow and very accurate withdrawal technique, but a few lesions can be recognized only using a scope able to visualize the back of the folds and of the flexures. Our data also suggest that a correct $\mathrm{WT}$, i. e. withdrawal technique, is a fundamental requisite when performing colonoscopy, independent of which endoscope is used, because when using the FUSE, it is also necessary to perform up and down tip movements to cover the mucosal surface in the vertical plane (the wide angle of view of FUSE pertains only to the lateral direction); moreover, effectively monitoring the three screens for exposed polyps can be difficult and time-consuming. Interestingly, ADR achieved by the FUSE in the preintervention phase did not differ from the postinterventional ADR obtained with the SFVE, a result that is likely to be important because the FUSE, exposing all the colonic mucosa faster and more efficiently than the SFVE, would potentially reduce WT duration without affecting accuracy.

This is not the first time that the FUSE has demonstrated promising results in comparison with a SFVE. In a previous tandem-colonoscopy study by Gralnek et al. [9], use of the FUSE resulted in a significantly lower adenoma miss rate in comparison to the SFVE. More recently, a randomized multicenter Italian study performed in the setting of the national CRC screening program was not able to demonstrate any statistically significant differences in ADR and advanced ADR between FUSE and SFVE in FIT-positive individuals [15]. This study was, however, performed on a very select population with an expected very high prevalence of neoplastic lesions, and endoscopists were likely to have maximized the diagnostic accuracy of each single technique to avoid relevant false-negative results. That fact may have minimized advantages of the FUSE over the SFVE. In addition, the detection rate for advanced neoplasia rather than ADR was the primary endpoint of the study.

Gralnek et al. found that most of the lesions missed by colonoscopy are diminutive polyps of the right colon [9]. A more accurate inspection of the right colon using retroflexion of the scope or segmental repetition of the withdrawal phase has been suggested to reduce the polyp miss rate [16]. These data are in accordance with our finding that optimizing WT may result in a significant increase in number of lesions detected in the right colon with the SFVE, while use of the FUSE is likely to offer only a very small (about $2 \%$ ) further increase in detection rate; conversely, in the left colon, optimization of WT is likely to carry a limited benefit when a SFVE is used, while use of the FUSE seemed to increase the detection rate. It is plausible that in a quite straight colon, such as the proximal one, rotation of the tip of the scope combined with in-out movements of tube insertion would allow a standard narrow view scope to cover the colon mucosa, while in the sigmoid colon, the very narrow curves and flexures would create several hidden areas that can be exposed only using a wider-angle view scope or a system able to flatten the folds and straighten the curvatures.

A recent study has demonstrated that unmonitored endoscopists may have poor adherence to the WT protocol, but that their WT increases (and also their ADR) when they are aware of being monitored [10]. This information has been reproduced in our study and has allowed to us to evaluate the effectiveness of instituting an adequate WT in improving accuracy of colonoscopy. Reduced adherence to WT recommendations seems to be a real problem in the real world [17], and some centers systematically monitor WT to increase endoscopists' adherence [18]. The physicians performing our study are all very expert endoscopists, who had performed more than 1000 investigations; they all were aware of the value of WT in improving accuracy of colonoscopy, and in previous monitored sessions, they showed a high adherence to the 6-minute withdrawal protocol. In spite of this, in the first part of the study, only about $22.9 \%$ of procedures were performed with a WT $>6$ minutes.

We have demonstrated that WT, i.e. attention that the endoscopists pay during scope retraction, was strictly related to the subjective perceived chance of finding a significant lesion at endoscopy. As a consequence, in phase 1, when endoscopists were unaware of being monitored, WT was longer when a first adenoma was detected during the procedure, as well as in patients undergoing colonoscopy for CRC screening; conversely, WT was shorter in cases of overt bleeding, an indication for which endoscopy is performed to look for grossly bleeding lesions. The clinical impact of such behavior has yet to be evaluated, but it is likely to be minimized by use of the FUSE. 
Our study design deserves some consideration. As a real-life study, patients were not randomized and assignment to undergo colonoscopy with one or the other scope was only determined by scope availability. The two groups were, however, comparable for demographic and clinical characteristics, which would have minimized risk of bias. In addition, randomization would not have ensured any blind comparison, as the endoscopist could not be masked to the type of scope used.

The study design allowed recruitment of patients undergoing colonoscopy for different indications. Thus, only $30 \%$ of patients were screened for colonoscopy, whereas the values of ADR are strictly defined. Instead, in our setting, heterogeneity of indications for colonoscopy makes it difficult to compare our results with ADRs of other studies, which may be a limitation of this study.

Overall in this study, both introduction and retraction times were longer for the FUSE in comparison with the SFVE, a fact that would suggest that endoscopists were not completely confident with using the FUSE. Prior to the study's start, a formal training was done and every endoscopist performed 10 investigations with the new instrument to get familiar with it. We could hypothesize that the learning curve for the FUSE may be longer even for expert endoscopists, but because insertion time for the FUSE was similar in the two phases of the study, it is more likely that characteristics of the FUSE system may be associated with some difficulties related to maneuverability and that the need to monitor three screens may result in expenditure of more time.

\section{Conclusion}

In conclusion, we have demonstrated that unmonitored endoscopists have suboptimal WT, and that WT increases when they are aware of being monitored. Optimizing WT is a fundamental requisite when performing colonoscopy, whichever endoscope is used. Even if used with adequate WT, standard scopes may be unable to visualize all lesions, and use of the FUSE may increase the number of lesions detected. While detection of proximal adenomas is more strictly related to application of a correct WT protocol, use of the FUSE may represent an important aid in detection of lesions in the distal colon.

\section{Competing interests}

EndoChoice provided the FUSE equipment for the study with no other involvement in the analysis of the data.
References

[1] Mandel JS, Church TR, Bond JH et al. The effect of fecal occult-blood screening on the incidence of colorectal cancer. N Engl J Med 2000; 343: $1603-1607$

[2] Jorgensen OD, Kronborg O, Fenger C. A randomised study of screening for colorectal cancer using faecal occult blood testing: results after 13 years and seven biennial screening rounds. Gut 2002; 50: $29-32$

[3] le Clercq CM, Bouwens MW, Rondagh EJ et al. Postcolonoscopy colorectal cancers are preventable: a population-based study. Gut 2014; 63: $957-963$

[4] Barclay RL, Vicari J], Doughty AS et al. Colonoscopic withdrawal times and adenoma detection during screening colonoscopy. N Engl J Med 2006; 355: $2533-2541$

[5] Rex DK. Colonoscopic withdrawal technique is associated with adenoma miss rates. Gastrointest Endosc 2000; 51: 33 - 36

[6] Lee RH, Tang RS, Muthusamy VR et al. Quality of colonoscopy withdrawal technique and variability in adenoma detection rates (with videos). Gastrointest Endosc 2011; 74: 128 - 134

[7] Rembacken B, Hassan C, Riemann JF et al. Quality in screening colonoscopy: position statement of the European Society of Gastrointestinal Endoscopy (ESGE). Endoscopy 2012; 44: 957- 968

[8] Sawhney MS, Cury MS, Neeman N et al. Effect of institution-wide policy of colonoscopy withdrawal time > or $=7$ minutes on polyp detection. Gastroenterology 2008; 135: 1892-1898

[9] Gralnek IM, Siersema PD, Halpern Z et al. Standard forward-viewing colonoscopy versus full-spectrum endoscopy: an international, multicentre, randomised, tandem colonoscopy trial. Lancet Oncol 2014; 15: $353-360$

[10] Vavricka SR, Sulz MC, Degen L et al. Monitoring colonoscopy withdrawal time significantly improves the adenoma detection rate and the performance of endoscopists. Endoscopy 2016; 48: 256-262

[11] Brand EC, Wallace MB. Strategies to increase adenoma detection rates. Curr Treat Options Gastroenterol 2017; 15: 184-212

[12] Simmons DT, Harewood GC, Baron TH et al. Impact of endoscopist withdrawal speed on polyp yield: implications for optimal colonoscopy withdrawal time. Aliment Pharmacol Ther 2006; 24: 965 - 971

[13] Dik VK, Gralnek IM, Segol O et al. Multicenter, randomized, tandem evaluation of EndoRings colonoscopy - results of the CLEVER study. Endoscopy 2015; 47: 1151-1158

[14] Moritz V, Bretthauer M, Ruud HK et al. Withdrawal time as a quality indicator for colonoscopy - a nationwide analysis. Endoscopy 2012; 44: $476-481$

[15] Hassan C, Senore C, Radaelli F et al. Full-spectrum (FUSE) versus standard forward-viewing colonoscopy in an organised colorectal cancer screening programme. Gut 2017; 66: 1949-1955

[16] Rex DK. How I approach retroflexion and prevention of right-sided colon cancer following colonoscopy. Am J Gastroenterol 2016; 111: $9-11$

[17] Butterly L, Robinson CM, Anderson JC et al. Serrated and adenomatous polyp detection increases with longer withdrawal time: results from the New Hampshire Colonoscopy Registry. Am J Gastroenterol 2014; 109: 417-426

[18] Barclay RL, Vicari J], Greenlaw RL. Effect of a time-dependent colonoscopic withdrawal protocol on adenoma detection during screening colonoscopy. Clin Gastroenterol Hepatol 2008; 6: 1091 - 1098 